\title{
Microstructure Evolution Simulation during Microextrusion at Elevated Temperature
}

\author{
S. CHEN ${ }^{1, a}$, F. CHEN ${ }^{1, b}$, L.J. ZHANG ${ }^{1, c}$, X.H. HUANG ${ }^{1, d}$, X.Y. DING ${ }^{1, e}$, \\ X.P. ZHANG ${ }^{1, f, *}$ \\ ${ }^{1}$ Department of Materials Science and Engineering, Nanjing University of Science and Technology, \\ Nanjing 210094, China \\ a592001575@qq.com, bchenf1990@163.com, ${ }^{c} 1146625530 @ q q . c o m,{ }^{d} 1522421091 @ q q . c o m$, \\ e18362961673@163.com, f, xpzhang@njust.edu.cn
}

${ }^{*}$ Corresponding author

Keywords: Microextrusion, Elevated Temperature, Microstructure Evolution, Finite Element Method.

\begin{abstract}
Controlling the microstructure in the final product is significant to metal hot forming at either macroscale or microscale. The microstructure evolution during the hot forming must be fundamentally understood in order to control the microstructure. Elevated temperature contributes to minimize the size-effects of microforming processes and also enlarge the forming limits by microforming. Recrystallization and grain growth during microextrusion at elevated temperature followed by air-cooling were studied using conventional recrystallization theory models and finite element methods. The results showed the microstructure distribution in the extruded billet can be rationally predicted based on the conventional recrystallization theory models. The simulation of the grain size distribution agreed with the experiment. A larger extrusion ratio, a faster extrusion speed and a refiner initial grain all help to obtain a smaller grain size and a more homogeneous grain distribution in the extruded billet.
\end{abstract}

\section{Introduction}

During the past decades, the development towards miniaturization of products and devices in industries, such as electronics, automobiles and healthcare, has increased the demand for metallic parts manufactured at microscale. The properties of a metal are strongly depended on its microstructural features. Thus, controlling the final microstructure distribution plays a significant role in hot metal forming, whether at either macroscale or microscale. The stochastic distribution of the grain characteristics leads to an inhomogeneous material behavior and causes an increased scatter of the process parameters. Elevated temperature contributes to minimize the size-effects at microforming processes and enlarge the micoforming limits [1,2]. The microstructure evolution simulation for microforming is still at its preliminary stage. Numerical simulation is the most accurate method to study the heat transfer, deformation behavior, recrystallization and grain growth process during the deformation. Microstructures simulation of microforming at room temperature using the crystal plasticity theory [3-5]. However, the microstructure experiences a complicated evolution during the hot forming. There are few studies on the recrystallization and the grain growth of microparts during the microforming at elevated temperature [6]. The effects of the microforming parameters on microstructure have also not been reported.

\section{Simulation details}

The microstructure evolution during the extrusion and subsequent air-cooling was simulated by finite element methods (FEM) using Deform 2D software. The size of the billet was $\varnothing 0.5 \times 0.5 \mathrm{~mm}$. The total displacement of the pressure stem was $90 \%$ of the initial height of the billet. After completing the microextrusion, the die and the pressure stem were removed, the billet was air cooled 
to a defined temperature. The material used was AMS 5704 steel, and its composition is listed in Table 1.The assumption for this simulation was to model only half a slice of the extrusion billet, starting from the centerline.

The microstructure evolution models are listed in Table 2. Different frictional coefficients $(0.10$, 0.3 and 0.7$)$, extrusion ratios $(1.56,2.04,2.78$ and 4$)$, extrusion speeds $(0.0005 \mathrm{~mm} / \mathrm{s}, 0.05 \mathrm{~mm} / \mathrm{s}, 0.5$ $\mathrm{mm} / \mathrm{s}$ and $2.5 \mathrm{~mm} / \mathrm{s})$, and initial grain sizes $(20 \mu \mathrm{m}, 40 \mu \mathrm{m}$ and $120 \mu \mathrm{m})$ were used for investigating the effects of the extrusion conditions and the initial grain size on the recrystallization and the grain growth. If no special instruction, extrusion ratio was 1.56 , extrusion speed was $0.5 \mathrm{~mm} / \mathrm{s}$, extrusion die angle was $45^{\circ}$, frictional coefficient was 0.10 , and initial grain size was $40 \mu \mathrm{m}$.

Tab. 1 Composition of the experimental material [wt. \%]

\begin{tabular}{|c|c|c|c|c|c|c|c|c|c|c|c|}
\hline $\mathrm{Al}$ & $\mathrm{B}$ & $\mathrm{C}$ & $\mathrm{Cr}$ & $\mathrm{Co}$ & $\mathrm{Fe}$ & $\mathrm{Mn}$ & Mo & $\mathrm{Ni}$ & $\mathrm{Si}$ & $\mathrm{Ti}$ & $\mathrm{Zr}$ \\
\hline 1.50 & 0.0060 & 0.080 & 19.0 & 13.5 & $\leq 2.0$ & $\leq 0.10$ & 4.30 & 56.0 & $\leq 0.15$ & 3.0 & 0.05 \\
\hline
\end{tabular}

Tab. 2 Microstructual evolution model used in Deform [7]

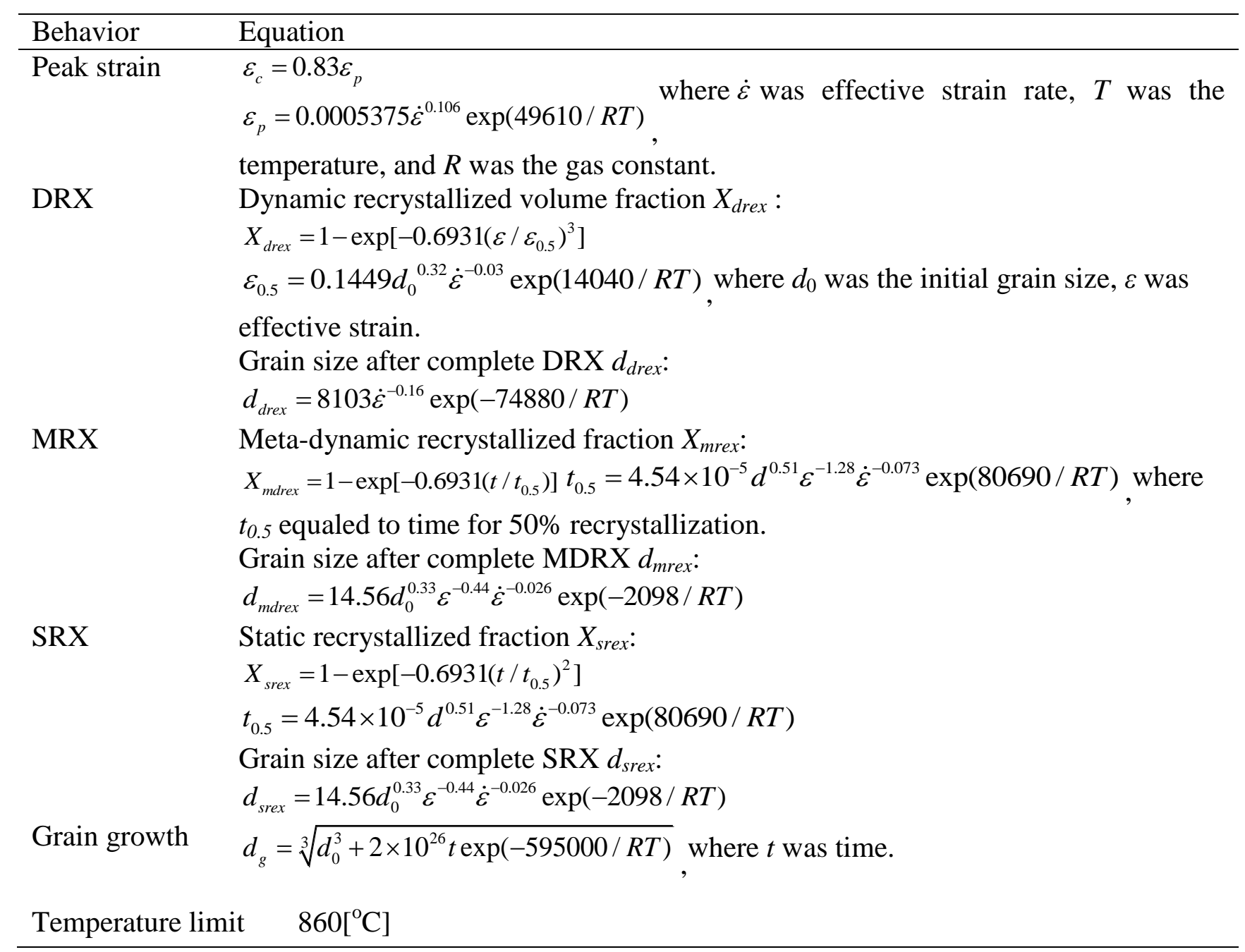

\section{Results and Discussion}

\section{Field-variables and Microstructure Distributions}

Xdrx, Xmdrx, X srx and grain size distribution in the extruded billet are shown in Figs.1a to d. There were three zones in the extruded billet (Fig. 1 a): Zone 1 is a deforming zone. Zone 2 is the 
deformed zone. There is a refined zone in the Zone 2, as shown in Fig. $1 \mathrm{~d}$. In addition, there was a zone without any severe deformation located at the top of the billet, which was labeled as Zone 3 . It was also noted that Xdrx and Xmdrx were small in Zone 3, while SRX occurred only in Zone 3.

Fig. 1d and 2a show that the grain size decreased and the equivalent strain increased from the centre to the surface of the extruded billet cross section. The selected position L1 is shown in Fig.1d. In addition, Xmdrx also decreased from the centre to the surface, while Xdrx increased until reached a peak value and then decreased. However, Xdrx near the surface of the billet increased with the distance from the centre, as shown in Fig.2b.

Fig. 2c and $\mathrm{d}$ show the typical microstructure evolution during the process. Dynamic recrystallization occurred firstly in Zone 2, followed by metadynamic recrystallization; Static recrystallization did not occur in this zone, as shown in Fig.2c. For Zone 3, static recrystallization occurred first and was followed by dynamic recrystallization, and finally metadynamic recrystallization, as shown in Fig.2d.
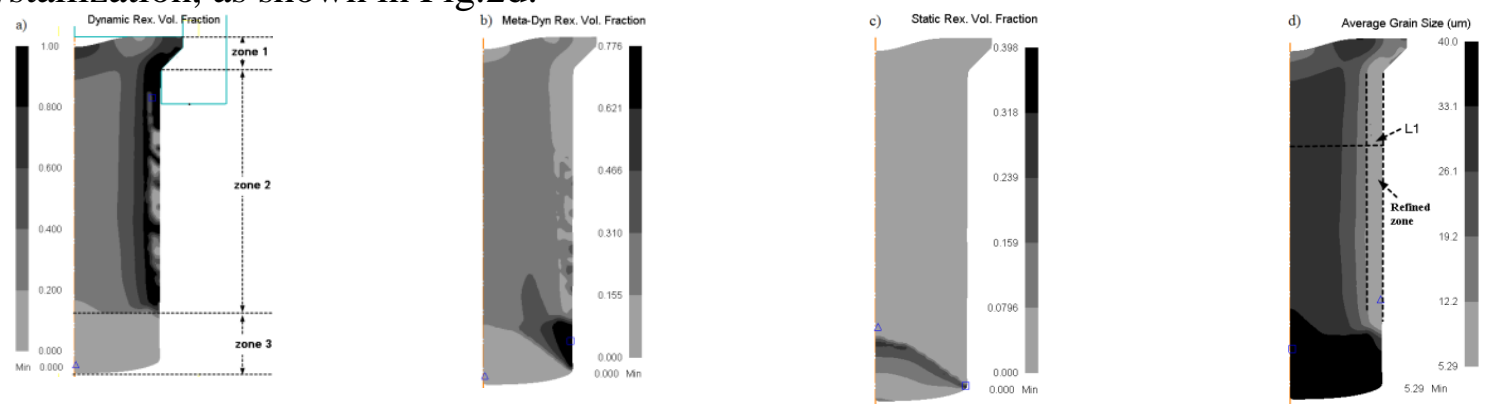

Fig. 1 Field-variables and microstructure distribution in the extruded billet. a) Xdrx, b) Xmdr , c) X srx, d) grain size

From Fig.1, it was also noted that the majority part of recrystallization volume fraction was Xdrx in Zone 2. Thus, metadynamic recrystallization and static recrystallization will not be discussed in the subsequent section.

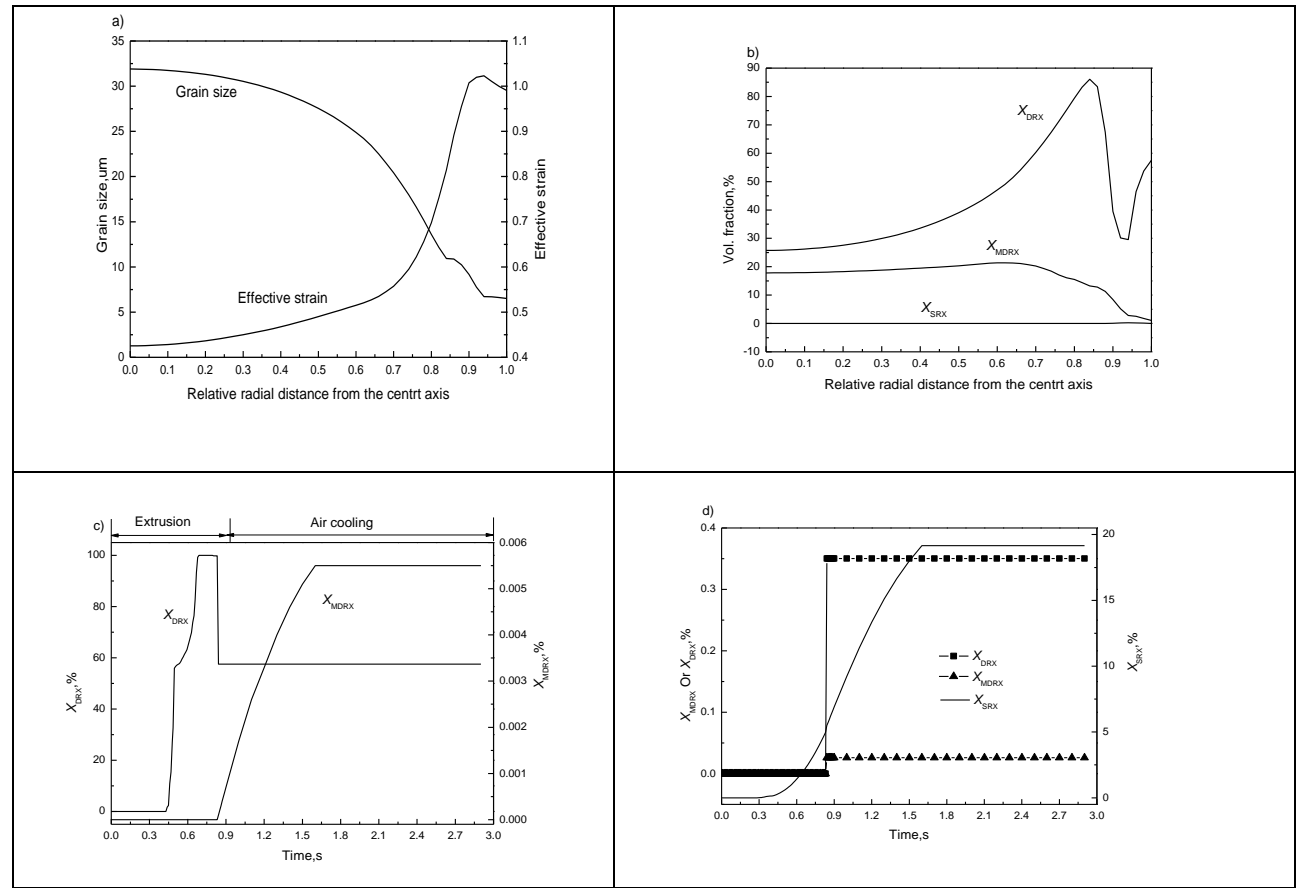

Fig. 2 Distribution of equivalent strain and the grain size along the section (L1) and microstructure evolution during the process. a) distribution of equivalent strain and the final grain size along the section, b) distribution of recrystallized volume fraction along the section, c) microstructure evolution with time in Zone 2, and d) microstructure evolution with time in Zone 3. 


\section{Effects of Frictional Coefficient}

Friction is one of the most important phenomena in metal forming process. Friction at the microscale is different from conventional friction because of the size effects [8]. As shown in Fig.3, it can be concluded that the effects of the frictional coefficient on the recrystallization and the final grain size were slight. Frictional coefficient affected the temperature rise during the microextrusion. In this work, the maximum temperature rise increased from $60 \mathrm{oC}$ to $80 \mathrm{oC}$ when the frictional coefficient increased from 0.1 to 0.7. Based on the models in Table 2, the peak strain decreased with the deformation temperature, while Xdrx, Xmrex, Xsrex, ddrex, dmrex and dsrex increased with them. Thus, the final grain size and the final relative width of the refined zone changed slightly with the frictional coefficient.

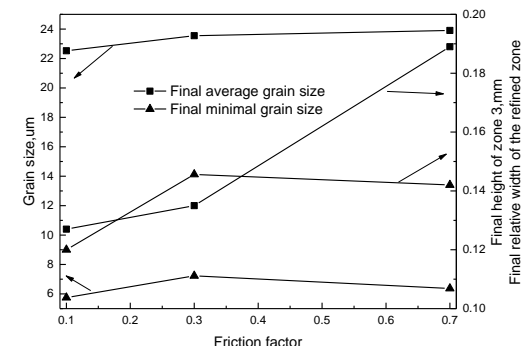

Fig. 3 Effects of frictional coefficient on the grain characteristics

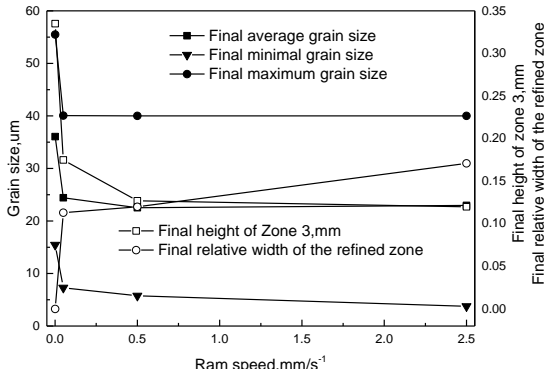

Fig. 4 Effects of extrusion speed on the grain characteristics

\section{Effects of Extrusion Speed}

The extrusion speed influences the strain rate and the extrusion time in the extruded billet. The strain rate increased with the extrusion speed. According to the models in Table 2, Xdrex, Xmrex and Xsrex increased and the final grain size decreased with the extrusion speed, as shown in Fig.4.

\section{Effects of Extrusion Ratio}

There was a large difference in the distributions of grain size and Xdrx in the extruded billet at different extrusion ratios are shown in Fig.1 and Fig.5. The final average grain size and the final height of Zone 3 decreased with the extrusion ratio. The width of the refined zone increased with the extrusion ratio.

The strain rate and the strain magnitude increased with the extrusion ratio. According to the models in Table 2, Xdrex, Xmrex and Xsrex increased and the final grain size decreased with the extrusion ratio. In addition, the peak strain increased with the strain rate, which becomes an obstacle for the occurrence of dynamic recrystallization. These contradictory effects of the extrusion ratio on the dynamic recrystallization lead to the "bamboo-shaped" distribution of Xdrex along the extrusion direction, as shown in Fig.4c.
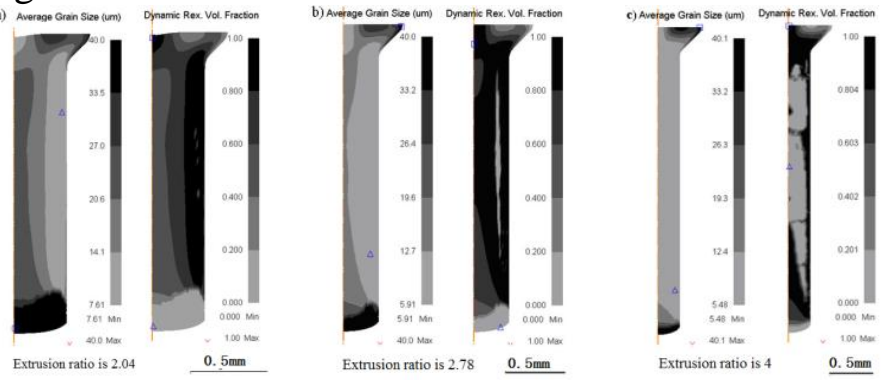

Fig. 5 Field-variables and microstructure distribution in the extruded billet at different extrusion ratios. a) extrusion ratio is $2.04, b$ ) extrusion ratio is 2.78 , and c) extrusion ratio is 4 


\section{Effects of Initial Grain Size}

As shown in Fig.6, Xdrx decreased with the initial grain size. The maximum Xdrx was only $77.2 \%$ when the initial grain size was $120 \mu \mathrm{m}$, and it reached $100 \%$ when the initial grain size was $20 \mu \mathrm{m}$. The decreased of Xdrx decreased the amount that is refined. The height of Zone 3 also increased with the initial grain size. These phenomena can also be explained using the models in Table 2 . The recrystallization grain size was proportional to the initial grain size, while the recrystallization volume fractions were inversely related to the initial grain size.

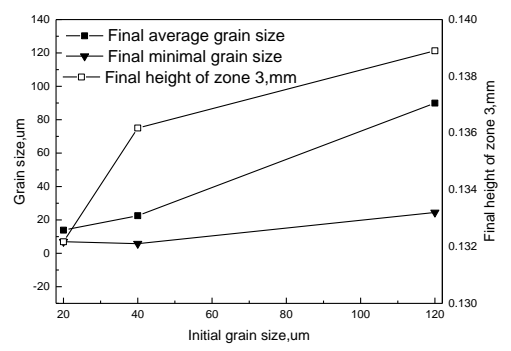

Fig. 6 Effects of initial grain size on the grain characteristics

From Figs. 1 to 6, the microstructure distributions in the extruded billet after the microxtrusion and subsequent air-cooling were predicted based on the conventional recrystallization theory models, and the simulations of the grain size distribution agreed with the experiments [9].

In general, the optimization goal for the microstructures after hot deformation is a small grain size and a homogeneous grain distribution [10] to improve the characteristics of the components produced. From the above results, the homogeneous grain distribution in the extruded billet can be determined using the final height of Zone 3 and the relative standard deviation of the final grain size. In addition, a larger extrusion ratio, a faster extrusion speed and a finer initial grain all help to obtain a smaller grain size and a more homogeneous grain distribution in the microextruded billet.

\section{Summary}

The microstructure distribution after microextrusion and subsequent air cooling can be predicted based on conventional recrystallization theory models. The simulation agreed with the experiment [9].The recrystallization and the grain size distributions in the extruded billet were inhomogeneous due to the inhomogeneous deformation. A larger extrusion ratio, a fast extrusion speed and a finer initial grain all help to obtain a smaller grain size and a more homogeneous grain distribution in the microextruded billet.

\section{Acknowledgement}

This work was supported by Jiangsu Qinglan Project; the Scientific Research Foundation for the Returned Overseas Chinese Scholars, State Education Ministry; the Fundamental Research Funds for the Central Universities, No. 30920140112008.

\section{Reference}

[1] B. Eichenhueller, E. Egerer and U. Engel: Int. J. Adv. Manuf. Technol. 33(2007), p. 119-124.

[2] N. Bontcheva, G. Petzov and L. Parashkevova: Comput. Mater. Sci. 38(2006), p. 83-89.

[3] E. Egerer and U. Engel: J. Manuf. Process. 6(2004), p. 1-6.

[4] S. Wang, W. Zhuang and D. Balint, J. Lin: Proceeded Eng 1 (2009), p. 75-78. 
[5] J. Cao, W. Zhuang, S. Wang, K.C. Ho, N. Zhang, J. Lin and T.A. Dean: J. Multis. Model. 1(2009), p. 107-124.

[6] X.T. Hong, F. Chen, F. Chen, W. Yu, B.R. Sang and X.P. Zhang: Adv. Mater. Res. 1004-1005 (2014), p. 158-162.

[7] Deform Manuals (Scientific Forming Technologies Corporation, 2011).

[8] A. Messner, U. Engel, R. Kals and F. Vollertsen: J. Mater. Process. Technol. 45(1994), p. 371-376.

[9] K.F. Zhang: Microforming Technology (Chemical Industry Press, Beijing 2008).

[10] J. Guan, G.C.Wang, T. Guo, L.B. Song and G.Q. Zhao: Mater. Sci. \& Eng. A 499 (2009), p. 304-308. 